\title{
TAMIZAJE NACIONAL UNIFICADO DE HIPOTIROIDISMO CONGÉNITO EN EL PERÚ: UN PROGRAMA INEXISTENTE
}

\author{
Lina Huerta-Sáenz ${ }^{1,2,3, a}$, Carlos Del Águila4,a, Oscar Espinoza ${ }^{4, a}$, Juan Falen-Boggiơ,a, Naim Mitre 1,3,a
}

\begin{abstract}
RESUMEN
El hipotiroidismo congénito $(\mathrm{HC})$ es la principal causa prevenible de retardo mental. La prevalencia de $\mathrm{HC}$ varía de acuerdo con la región geográfica y la población racial. En el hemisferio norte, la incidencia es 1:4000 recién nacidos vivos. En Latinoamérica, la incidencia varía debido a la heterogeneidad racial/étnica y al nivel de desarrollo. En el Perú, un estudio realizado en 1984 describió esta prevalencia como 1:1250. En el 2007, esta prevalencia fue reportada como 1:1638 por el Instituto Nacional Materno Perinatal. Un reciente estudio del Instituto Nacional de Salud del Niño durante 1995-2005, describió la edad promedio de diagnóstico de HC como 5,9 meses +/- 5,28. Esta tardía edad de diagnóstico sugiere la poca eficiencia de los actuales programas de tamizaje. Todo niño peruano tiene derecho a recibir diagnóstico oportuno y tratamiento de $\mathrm{HC}$ y es el Estado Peruano, el responsable principal de asegurar que esta meta se cumpla.
\end{abstract}

Palabras clave: Hipotiroidismo congénito; Tamizaje neonatal; Niño, Perú (fuente: DeCS BIREME).

\section{NATIONAL CONGENITAL HYPOTHYROIDISM SCREENING IN PERU: A BROKEN PROGRAM}

\begin{abstract}
Congenital hypothyroidism $(\mathrm{CH})$ is the most important cause of preventable mental retardation. The prevalence of $\mathrm{CH}$ varies by geographic region, race and ethnicity. In the countries of the Northern hemisphere, the prevalence has been reported as 1:4,000 live newborns. The prevalence is remarkably different among the countries of Latin America not only because of their different races and ethnicities but also because of the heterogeneous social-economic development. The prevalence of $\mathrm{CH}$ in 1984 in Peru was reported as 1:1250. In 2007, the reported incidence by the Instituto Nacional Materno Perinatal was 1:1638. A recent retrospective study performed by the Instituto Nacional de Salud del Niño in Lima, Peru described the average age of diagnosis of $\mathrm{CH}$ as 5,9 months $+/-5,28$. This late age of $\mathrm{CH}$ diagnosis certainly suggests the poor efficiency of the current neonatal $\mathrm{CH}$ screening programs in Peru. Every Peruvian infant deserves a timely newborn screening and treatment for $\mathrm{CH}$. The Peruvian government is responsible for ensuring this mandatory goal is achieved promptly.
\end{abstract}

Key words: Congenital hypothyroidism; Neonatal screening; Child, Peru (source: MeSH NLM).

\section{INTRODUCCIÓN}

El hipotiroidismo congénito $(\mathrm{HC})$ es la causa más común de retardo mental prevenible en la población pediátrica. La prevalencia mundial de $\mathrm{HC}$ es reportada como 1: 4000 nacidos vivos en países desarrollados del hemisferio norte (1). A nivel latinoamericano, un estudio de prevalencia de $\mathrm{HC}$ en Argentina durante los años 1997-2010 describió 1:2367 - 1:3108 nacidos vivos ${ }^{(2)}$. En el Perú, la prevalencia varía de acuerdo al sistema de salud ejecutor del tamizaje debido a la inexistencia de un programa nacional unificado. En Perú existen pocos estudios publicados al respecto, sin embargo, uno de los artículos más recientes reportó edad tardía de diagnóstico $(5,9$ meses $\pm 5,3)$ sugiriendo la poca eficiencia de los actuales programas de tamizaje neonatal de $\mathrm{HC}{ }^{(3)}$.

El objetivo de este artículo es hacer una revisión del desarrollo del programa de tamizaje neonatal de

\footnotetext{
Children’s Mercy Hospital, Missouri, Kansas, EE. UU.

University of Kansas Medical Center. Kansas, EE. UU.

University of Missouri-Kansas City, Missouri, EE. UU.

Instituto Nacional de Salud del Niño. Servicio de Endocrinología Pediátrica. Lima, Perú.

Endocrinólogo pediatra

Recibido: : 26-01-15 Aprobado: 13-05-15
}

Citar como: Huerta-Sáenz L, Del Águila C, Espinoza O, Falen-Boggio J, Mitre N. Tamizaje nacional unificado de hipotiroidismo congénito en el Perú: un programa inexistente. Rev Peru Med Exp Salud Publica. 2015;32(3):579-85. 
hipotiroidismo congénito en el Perú; comparar el actual desarrollo de Perú en este programa con relación a otros países del continente, sensibilizar a la población y a los profesionales de salud respecto a la importancia de un eficiente programa nacional de tamizaje de $\mathrm{HC}$ $\mathrm{y}$, debido al impacto en la población, sugerir posibles estrategias para mejorar la tardía edad de diagnóstico de $\mathrm{HC}$ en el Perú.

\section{ASPECTOS HISTORICOS DEL TAMIZAJE NEONATAL DE HIPOTIROIDISMO CONGÉNITO EN EL PERÚ}

La primera iniciativa legal para iniciar el tamizaje nacional de HC surgió en 1997 a través de la Resolución Ministerial 494-97-SA/DM en la que se resolvió Declarar el tamizaje para el diagnóstico de hipotiroidismo congénito de uso necesario en todos los servicios de neonatología a nivel nacional ${ }^{(4)}$. Este mismo año, el Instituto Nacional de Salud del Niño (INSN) inauguró el primer laboratorio de tamizaje neonatal de $\mathrm{HC}$ en el Perú, con un equipo de última generación donado por la JICA (Japan International Cooperation Agency) como parte de un programa de entrenamiento técnico a profesionales de países en desarrollo ${ }^{(5)}$. Este curso anual de tres meses fue realizado por el instituto de Salud Pública de la ciudad de Sapporo, Japón, en 1991, gracias al programa de cooperación internacional de la JICA ${ }^{(6)}$. Desafortunadamente, este proyecto piloto no contó con financiamiento del Estado Peruano, y solo se pudo realizar un estudio piloto financiado por los padres en un hospital materno infantil de Lima ${ }^{(5)}$.

El Hospital Edgardo Rebagliati Martins, en el año 2002, fue el primer establecimiento de salud en iniciar el tamizaje neonatal de $\mathrm{HC}$, habiendo implementado esta estrategia a nivel nacional para el sistema de EsSalud. De acuerdo con un reporte publicado en el 2013 por Galán-Rodas, EsSalud tenía una cobertura del 100\% de recién nacidos para $\mathrm{HC}$, hiperplasia suprarrenal congénita, fenilcetonuria y galactosemia, habiendo tamizado a 476287 neonatos hasta junio de $2002{ }^{(7)}$.

En octubre de 2003, el Instituto Nacional Materno Perinatal (INMPN), instituto de referencia nacional para atención obstétrica en Lima (Perú), creó su propio programa de tamizaje neonatal iniciando un programa piloto desde octubre 2003 a febrero 2004 con la detección de $\mathrm{HC}$, hiperplasia suprarrenal congénita, fenilcetonuria y galactosemia. De acuerdo con el reporte publicado por el INMPN en su página web institucional, desde marzo 2004 hasta abril 2014 la cobertura del programa para los niños nacidos en dicho centro fue de $98,8 \%{ }^{\left({ }^{(8)}\right.}$. Este mismo artículo describió que el Ministerio de Salud de
Perú (MINSA): No cuenta con los recursos de estructura necesarios para implementar una estrategia similar a nivel nacional y sugiere la creación de 4 a 5 centros de referencia nacional para ser responsables de este tamizaje unificado.

La segunda iniciativa fue la publicación de la Norma de Atención Integral del Niño y la Niña NTS 040/ MINSA/DGSP-V.01, con Resolución Ministerial el 20 de marzo de 2006. Esta norma señaló el carácter de obligatoriedad del tamizaje de $\mathrm{HC}$ en el Perú por primera vez, describiendo que: El tamizaje de TSH se realizará en el $100 \%$ de recién nacidos al momento de alta o como máximo a las 60 horas de vida a través de una muestra de sangre de talón y su correspondiente recolección en papel de filtro ${ }^{(9)}$.

\section{DIAGNÓSTICO DE HIPOTIROIDISMO CONGÉNITO}

El diagnóstico de $\mathrm{HC}$ se realiza mediante pruebas de función hormonal tiroidea, específicamente mediante la medición plasmática de hormona estimulante de la tiroides (TSH) y/o tiroxina libre (T4 libre). Ambas pruebas deben ser recolectadas después de las primeras 48 horas de vida, para evitar obtener valores fisiológicos elevados de TSH o T4 libre, debido al incremento fisiológico esperado después del nacimiento ${ }^{(10)}$.

Antes de obtener muestras plasmáticas del neonato para confirmación de $\mathrm{HC}$, se debe realizar el respectivo tamizaje neonatal de $\mathrm{HC}$ en papel del filtro, con sangre obtenida del talón. La muestra obtenida en papel de filtro debe ser recolectada después de las primeras 24 horas de vida, idealmente (11). En algunos países, muestras sanguíneas del cordón umbilical son utilizadas también como prueba de tamizaje. Los valores de las hormonas tiroideas obtenidas a través de muestras de papel de filtro pueden reportar TSH, T4 libre o T4 total dependiendo del requerimiento de cada programa. Los valores normales deben ser definidos de acuerdo con cada población.

Países como Canadá, Japón, Australia y en Europa, utilizan TSH como único examen para fines de tamizaje neonatal de $\mathrm{HC}{ }^{(10)}$, pero en otros países tales como Puerto Rico y algunos estados de los Estados Unidos de América (EUA) se realizan ambas pruebas (TSH y T4 libre) ${ }^{(10)}$. Resultados de TSH elevado y/o T4 libre baja o normal en la prueba de tamizaje sugiere HC, y el diagnóstico debe ser confirmado mediante la recolección de TSH y T4 libre en suero plasmático, si estos son anormales, el tratamiento hormonal tiroideo debe ser iniciado inmediatamente. En EUA, todos los recién nacidos reciben tamizaje neonatal para $\mathrm{HC}$ antes 
de recibir el alta hospitalaria. Algunos estados también realizan doble tamizaje: 1 ). Tamizaje inicial entre los 2 a 4 días de vida, y 2). Segundo tamizaje a las 2 semanas de vida, para detectar posibles casos de incremento leve o tardío de la TSH ${ }^{(12)}$.

Los valores de referencia "normales" deben ser obtenidos sobre la base de técnicas de normalización de laboratorios especializados en exámenes hormonales, y deben considerar también la edad específica del paciente, debido a que la concentración de TSH suele ser mayor en las primeras semanas y meses de vida. Existen tablas estandarizadas de valores normales de TSH, T4 libre, T3 y T4 total previamente publicadas y validadas ${ }^{(10)}$.

En febrero de 2014, la Sociedad Europea de Endocrinología Pediátrica publicó nuevas guías de consenso para el tamizaje, el diagnóstico y el tratamiento de HC. De acuerdo con estas, si la prueba de TSH en el tamizaje neonatal es igual o mayor a $40 \mathrm{mU} / \mathrm{L}$ se recomienda iniciar tratamiento tan pronto la prueba sanguínea confirmatoria sea recolectada, al menos que los resultados de función tiroidea estén disponibles el mismo día de la notificación de los resultados ${ }^{(13)}$.

\section{IMPORTANCIA DEL DIAGNÓSTICO OPORTUNO DE HIPOTIROIDISMO CONGÉNITO}

La razón primordial de la necesidad de tratamiento inmediato en casos confirmados de $\mathrm{HC}$ se debe a que en las etapas tempranas del desarrollo (los primeros 3 años de vida) el cerebro tiene dependencia crítica de la hormona tiroidea ${ }^{(1)}$. La demora en el tratamiento del HC puede ocasionar el deterioro de la función cerebral, así como pérdida del coeficiente intelectual y/o alteración del desarrollo psicomotor normal ${ }^{(14)}$.

Considerando que los síntomas de $\mathrm{HC}$ son inespecíficos, especialmente durante las etapas tempranas de la vida, la estrategia más exitosa para identificar $\mathrm{HC}$ antes de que la hipotiroxinemia ocasione consecuencias irreversibles es obteniendo pruebas de función tiroidea.

En 1986, el Boletín Médico del Hospital Infantil de México publicó una escala clínica creada por BlancoLópez para la evaluación de pacientes con sospecha de $\mathrm{HC}$ basada en el estudio previo de Letarte ${ }^{(15)}$. Esta escala clínica describía síntomas y signos tales como: piel seca, hipotonía, fontanelas amplias, estreñimiento, ictericia prolongada, entre otros signos/síntomas adicionales como sugestivos de $\mathrm{HC}$. El puntaje máximo era 21, y se recomendaba sospechar HC si el puntaje del paciente era igual o mayor a 4. Durante los años
80, cuando la mayoría de países de Latinoamérica no disponía de tecnología de laboratorio adecuada para realizar tamizaje neonatal de $\mathrm{HC}$ ni de pruebas bioquímicas de función tiroidea, esta escala clínica colaboró con la identificación de pacientes con síntomas y signos compatibles de HC. Actualmente, este tipo de escalas son poco confiables para realizar un diagnóstico oportuno de HC. Los infantes con HC suelen presentar niveles anormales de TSH mucho antes de presentar síntomas clínicos.

Durante los años de 1961-1963, países como Canadá, Inglaterra, Estados Unidos, Japón, entre otros, entendieron la importancia de iniciar políticas nacionales de tamizaje neonatal para identificar tempranamente patologías en recién nacidos que, sin tratamiento oportuno, desarrollarían graves problemas de salud tales como retardo mental, o daños neurológicos y metabólicos severos con secuelas de discapacidad. El primer programa de tamizaje neonatal para $\mathrm{HC}$ fue creado en Canadá en 1974 y posteriormente en EUA en 1975, después del éxito del programa piloto realizado en Pittsburgh (Pennsylvania) (16). Gracias al inicio del programa de tamizaje neonatal de $\mathrm{HC}$ en EUA, la identificación de neonatos con $\mathrm{HC}$ en este país se incrementó progresivamente de 1:4094 en 1987, a 1:2372 en el año 2002 (17).

\section{PROGRAMAS DE TAMIZAJE NEONATAL DE HIPOTIROIDISMO CONGÉNITO}

\section{TAMIZAJE NEONATAL DE HIPOTIROIDISMO CONGÉNITO EN LOS ESTADOS UNIDOS DE AMÉRICA}

En el año 2006, la Academia Americana de Pediatría (AAP) publicó guías actualizadas para el tamizaje neonatal y tratamiento de $\mathrm{HC}{ }^{(18)}$. La AAP no solamente describe las razones por las cuales un programa de tamizaje neonatal para $\mathrm{HC}$ requiere ser "eficiente" para poder ser exitoso, sino también propone guías clínicas específicas para tomar decisiones terapéuticas en función a niveles de TSH y T4 libres, incluyendo las controversias de tratamiento para $\mathrm{TSH}$ limítrofe entre 6-10 mU/L. Una sección muy importante es la que describe el proceso de notificación de resultados y el establecimiento del seguimiento del tamizaje anormal. De acuerdo con estas guías, el resultado del tamizaje de $\mathrm{HC}$ debe ser notificado al hospital o al médico que ordenó la prueba de tamizaje neonatal. De forma tal que estableciendo las guías para el proceso de seguimiento, se garantiza que el paciente con tamizaje anormal sea notificado prontamente y empiece un tratamiento oportuno. Se establece, también, la "responsabilidad 
de notificación" al médico que prescribió el tamizaje de HC y, de no poder este ser ubicado, la responsabilidad final recae en la institución ejecutora del tamizaje. Esta estrategia asegura el seguimiento y tratamiento oportuno de $\mathrm{HC}$.

\section{TAMIZAJE NEONATAL DE HIPOTIROIDISMO CONGÉNITO EN EUROPA}

Como se describió previamente, la Sociedad Europea de Endocrinología Pediátrica publicó recientemente un consenso de guías clínicas para tamizaje, diagnóstico y tratamiento de HC. Estas guías, a diferencia de las guías de la AAP, enfatizan no solo la importancia del tamizaje neonatal "obligatorio" para $\mathrm{HC}$, sino también enfatizan la importancia de consejería genética en caso haya historia familiar de enfermedad tiroidea congénita o de malformaciones congénitas asociadas, la importancia de indicar terapia de reemplazo hormonal tiroideo en casos de hipotiroidismo transitorio y remarcan la importancia de la evaluación continua del desarrollo neurológico y neurosensorial, específicamente el test de audición y monitoreo de retraso del lenguaje. Estas dos patologías son más frecuentes en infantes con $\mathrm{HC}{ }^{(13)}$.

\section{TAMIZAJE NEONATAL DE HIPOTIROIDISMO CONGÉNITO EN AMÉRICA LATINA}

En Latinoamérica, el estado de los actuales programas de tamizaje neonatal incluyendo el de $\mathrm{HC}$ es variado y de remarcable contraste. En el año 2007, Borrajo publicó un artículo sobre el estado de los programas de tamizaje neonatal en Latinoamérica a principios del siglo XXI y describió con detalle la diversa heterogeneidad cultural, étnica y demográfica, así como los diferentes sistemas de salud responsables del tamizaje neonatal en la mayoría de nuestros países latinoamericanos (19). Estas diferentes características en modalidad y ejecución de los programas nacionales de tamizaje lo llevaron a clasificar a los países de Latinoamérica en cinco diferentes grupos:

Grupo l: Cuba, Costa Rica, Chile y Uruguay; más avanzado nivel de desarrollo, cobertura nacional al $100 \%$, y el Gobierno es el responsable directo del tamizaje, el tratamiento y monitoreo.

Grupo II: Brasil, México y Argentina; cobertura nacional al $60-80 \%$, el sector privado es el principal responsable de la ejecución y el financiamiento del programa.

Grupo III: Colombia, Paraguay y Venezuela; iniciaron tamizaje en 1999.

Grupo IV: Nicaragua y Perú; iniciaron políticas nacionales a partir del 2005, y la cobertura nacional es solo de 4-6\%.
Grupo V: Guatemala, Republica Dominicana, Bolivia, Panamá y Ecuador; no tenían programas nacionales de tamizaje neonatal y las pruebas de tamizaje solo se realizaban en el sector privado con coberturas por debajo del $1 \%$.

Grupo VI: El Salvador, Honduras y Haití; las actividades de tamizaje neonatal eran casi inexistentes.

Estos grupos de desarrollo de programas neonatales de tamizaje, en orden numérico de menor a mayor, guardan correlación con el grado de avance económico de estos países. En este mismo artículo se describió que el Perú tenía una baja cobertura nacional de tamizaje neonatal de solo $3,9 \%$, y el soporte económico del programa recaía parcialmente en el gobierno central y en el sistema de seguro social para obreros y empleados (EsSalud), con normas legales específicas para el programa de tamizaje neonatal de $\mathrm{HC}$ en el año 2005. Esta baja cobertura en el Perú estaba abismalmente lejos de la cobertura nacional reportada por Argentina en el mismo año (64\%).

\section{TAMIZAJE NEONATAL DE HIPOTIROIDISMO CONGÉNITO EN EL PERÚ}

En Perú, la norma legal mandatoria para realización de tamizaje neonatal de hipotiroidismo congénito existe desde el 2006, como se describió anteriormente en la sección de aspectos históricos, pero no existe un programa nacional de tamizaje de $\mathrm{HC}$ que integre a todos los subsistemas de salud existentes para ejecutar el tamizaje de hipotiroidismo congénito. Por ende, no existe un organismo vigilante que verifique metas y medición de éxito del programa ${ }^{(9)}$, porque cada pequeño sistema realiza tamizaje de $\mathrm{HC}$ independientemente. Estos cuatro subsistemas fraccionados de salud existentes en nuestro país 1). EsSalud; 2). El Ministerio de Salud (MINSA); 3). La Sanidad de las Fuerzas Armadas, Policiales y Marina de Guerra, y el 4). El sistema privado de Salud, deberían ser monitorizados por un único organismo controlador gubernamental para garantizar que la cobertura del programa alcance un $100 \%$ a nivel nacional y no solo a nivel intrainstitucional. Lograr la homogenización del programa de tamizaje neonatal de $\mathrm{HC}$ en el sistema de salud peruano es ciertamente un desafío mayor, pero superar este desafío es mandatorio si se desea tener un programa nacional de tamizaje unificado.

Otros aspectos necesarios para garantizar el éxito del programa nacional incluyen la utilización de la técnica de laboratorio correcta, el proceso de control de valores normales de TSH y T4 libre, el proceso de notificación de resultados a las familias del paciente, y la confirmación de inicio de tratamiento inmediato después de notificación de resultados. 
Idealmente, todos estos infantes deberían tener visitas de seguimiento con el especialista. Considerando que nuestro país no tiene suficiente número de pediatras endocrinólogos, una de las alternativas es seguir incluyendo endocrinología pediátrica como una rotación mandatoria del residentado de Pediatría, incluir esta rotación también en la residencia de medicina familiar y en el entrenamiento anual de los médicos serumistas (medico-cirujano general, recién egresado de la escuela de medicina, realizando un año de servicio de atención de salud para pobladores de zonas geográficas con pocos desarrollo en el Perú). Todas estas etapas requieren un planeamiento estratégico y el trabajo conjunto de estos cuatro subsistemas de salud. Es importante que el programa nacional de tamizaje de $\mathrm{HC}$ permanezca vigente independientemente del Gobierno y/o el partido político vigente.

\section{TAMIZAJE NACIONAL UNIFICADO DE HIPOTIROIDISMO CONGENITO EN EL PERÚ: UNA PRIORIDAD NACIONAL}

La definición de tamizaje nacional unificado de $\mathrm{HC}$ describe un programa nacional integrado de tamizaje de $\mathrm{HC}$, en el cual cada subsistema de salud existente en el Perú notifique con carácter mandatorio a la oficina nacional especifica (los autores sugieren crear esta oficina dentro del ministerio de salud) los resultados del tamizaje de $\mathrm{HC}$ con el propósito de vigilancia y supervisión nacional. Esta oficina nacional sería el ente representativo del Estado peruano responsable de entrenar al personal de laboratorio encargado de procesar las muestras de papel de filtro en cada oficina responsable de cada subsistema de salud, de forma tal que exista homogenización en el proceso de laboratorio del tamizaje (técnica similar de procesamiento). Creemos necesaria también la existencia de laboratorios regionales vigilados por un laboratorio central. El personal de cada uno de estos laboratorios recibiría entrenamiento y equipamiento específico para el proceso de tamizaje por solo una oficina nacional reguladora.

De similar forma, cada subsistema de salud tendría un líder responsable del programa de tamizaje de $\mathrm{HC}$, el cual formaría parte del grupo nacional de expertos liderando el programa nacional unificado en el país. El programa de tamizaje nacional unificado podría iniciarse con un programa piloto y los resultados de este, se medirían para extensión directa a un programa nacional de tamizaje de HC. Si este programa es exitoso, se podría, posteriormente, considerar como ejemplo para otros tamizajes neonatales.
Sugerimos considerar los siguientes pasos adicionales en este proceso de diagnóstico de $\mathrm{HC}$ :

a) Realización de tamizaje de HC utilizando similar técnica en todos los laboratorios aprobados de cada subsistema de salud de Perú.

b) Notificación de resultados a la oficina regulatoria de Perú.

c) La oficina regulatoria sería responsable de la comunicación de resultados a la familia y/o institución donde se realizó el tamizaje, además de ser responsable de proporcionar recomendaciones de tratamiento específicas, y coordinar una visita de seguimiento de $\mathrm{HC}$ con el profesional entrenado dentro de las 2 -4 semanas siguientes de inicio de tratamiento.

Los autores consideramos que la construcción de un programa nacional unificado de tamizaje de $\mathrm{HC}$ es una prioridad nacional. Sin embargo, las opiniones deben basarse en evidencias. La siguiente pregunta es: ¿cuál es la prevalencia nacional de hipotiroidismo congénito en el Perú?

El primer reporte publicado al respecto fue en 1984 por Rojas, Garmendia et al. La prevalencia de HC fue descrita como 1:1250 ${ }^{(20)}$. Este estudio se realizó en una muestra de 1254 recién nacidos vivos en la Maternidad de Lima (actualmente denominado Instituto Nacional Materno Perinatal - INMPN) y en el hospital Dos de Mayo durante los meses de julio a agosto de 1983. Veinte años después de la publicación de la primera resolución ministerial al respecto en el Perú, el INMPN describió una incidencia de 1:1638 nacidos vivos en el año $2007^{(21)}$.

Si consideramos la edad de diagnóstico y de tratamiento de HC como una variable indirecta de medición del éxito del programa de tamizaje de $\mathrm{HC}$, podríamos concluir cuán exitoso es el programa. Los primeros estudios al respecto fueron descritos por el servicio de Endocrinología Pediátrica del Instituto Nacional de Salud del Niño (INSN) en una cohorte estudiada entre 19811990. De acuerdo al estudio realizado por Del Águila et al., la edad promedio de diagnóstico clínico de $\mathrm{HC}$ fue de 17 meses de edad ${ }^{(22)}$. Esta cifra fue realmente alarmante. Un estudio posterior en la misma institución en pacientes del mismo servicio atendidos entre 1995 -2005 reportó una muestra inicial de 247 historias clínicas con presunto diagnóstico de HC. La población final del estudio solo incluyó 37 de estas historias clínicas, debido a criterios de inclusión específicos, y en esta muestra la edad promedio de diagnóstico de HC fue de 5,9 meses $\pm 5,3^{(3)}$. Estos importantes datos pertenecen al INSN, centro de referencia nacional para la población pediátrica del MINSA. 
Ciertamente, la edad promedio de diagnóstico pareciera haber mejorado en el período de 1990-2005. Considerando que la primera norma regulatoria para tamizaje de $\mathrm{HC}$ fue publicada en 1997, esto podría haber influido en la mejora de la edad media de diagnóstico de $\mathrm{HC}$ y también el mayor entrenamiento de pediatras en el diagnóstico de HC. Sin embargo, es lamentable que la referencia de estos pacientes al INSN para diagnóstico de HC haya sido tardía

El diagnóstico y tratamiento oportuno de $\mathrm{HC}$ implica el inicio de tratamiento inmediatamente después del diagnóstico, pero idealmente antes de las primeras dos semanas de vida, con el objetivo de normalizar la hormona T4 libre ${ }^{(13)}$. Podemos concluir que, a pesar de haber mejorado la edad de diagnóstico clínico de $\mathrm{HC}$ en los últimos 20 años, Perú está aún lejos de la meta de prevención de consecuencias irreparables debido al diagnóstico tardío de $\mathrm{HC}$. El diagnóstico oportuno, el inicio de tratamiento inmediato y la rápida normalización de las pruebas tiroideas son requeridas para obtener resultados neurológicos óptimos en HC.

Un estudio de Selva, La Franchi et al. comparó el neurodesarrollo de pacientes con $\mathrm{HC}$; específicamente el nivel cognitivo, el éxito académico, la atención y el comportamiento de tres grupos de pacientes. Cada grupo de tratamiento recibió diferente dosis de reemplazo de hormona tiroidea, y las pruebas de función tiroideas se normalizaron en diferentes momentos. Aquellos pacientes cuya normalización de hormonas tiroideas demoró más, presentaron mayor frecuencia de retraso del habla en comparación con aquellos que normalizaron hormonas tiroideas más rápidamente ${ }^{(23)}$.

En el Perú, el Instituto Nacional de Estadística e Informática (INEI) reportó 794040 nacimientos

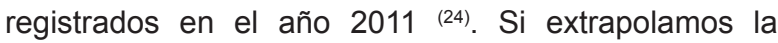
incidencia de HC previamente descrita por el INMP (1:1638), cada año nacerán 485 infantes con HC, y de no recibir diagnóstico y tratamiento oportuno, 485 infantes serán diagnosticados posteriormente con retardo mental, déficits neurocognitivos y/o neurosensoriales. Nuestras deficiencias en el manejo nacional de este problema continuarán ocasionando múltiples generaciones de peruanos con retardo mental y discapacidad, al menos que resolvamos el problema pronto.

Lograr una cobertura nacional de diagnóstico de HC y tratamiento oportuno al $100 \%$ requerirá muchos años de esfuerzo conjunto, pero podremos llegar a la meta si este problema es considerado una prioridad nacional. Esto se logrará no solo difundiendo conocimiento a los profesionales de salud, es decir, a los estudiantes de Medicina, médicos serumistas, médicos generales, residentes, pediatras, ginecólogos-obstetras, enfermeras, obstetrices, etc., sino también informando a la población de este problema y sus derechos en salud.

\section{CONCLUSIONES}

Los ciudadanos peruanos de todos los estratos sociales merecen acceder a un programa nacional de tamizaje neonatal de $\mathrm{HC}$, y merecen también la oportunidad de recibir diagnóstico temprano e inicio de tratamiento oportuno a fin de evitar retardo mental prevenible. Construir un programa nacional unificado de tamizaje, diagnóstico y tratamiento de $\mathrm{HC}$ es ciertamente una meta alta, pero definitivamente necesaria para poder avanzar como país.

Todo niño peruano, independientemente de su lugar de procedencia, de su aseguramiento en salud, de su estrato social o de su grupo étnico, merece la oportunidad de desarrollar todo su potencial neurocognitivo. El Estado peruano, como ente regulador nacional de la salud de todos los peruanos, es el principal responsable de ejecutar esta meta, y el MINSA, la institución responsable de vigilar que esta meta se cumpla.

Contribuciones de autoría: LHS realizo la concepción y diseño del artículo, y redactó la versión inicial y final del artículo. La versión final del artículo tuvo revisión crítica y aprobación final de todos los autores. NM realizó la revisión crítica de la versión final numerosas veces. LHS y NM redactaron la versión en inglés del resumen.

Fuentes de financiamiento: autofinanciado.

Conflictos de interés: los autores declaran no tener ningún conflicto de interés.

\section{REFERENCIAS BIBLIOGRÁFICAS}

1. LaFranchi SH, Austin J. How should we be treating children with congenital hypothyroidism?. J Pediatr Endocrinol Metab. May 2007;20(5):559-78.

2. Chiesa A, Prieto L, Mendez V, Papendieck P, Calcagno Mde L, Gruneiro-Papendieck L. Prevalence and etiology of congenital hypothyroidism detected through an Argentine neonatal screening program (1997-2010). Horm
Res Paediatr. 2013;80(3):185-92. doi: 10.1159/000354409.

3. Tovar Rodriguez N, Rojas Hernández A, Torres Matta F, Susaya Campos R, Del Aguila Villar C, Falen Boggio JM. Edad de diagnóstico clínico del hipotiroideo congénito: Veinte años después. Rev Peru Pediatr. 2014;67(1):17-21.

4. Peru, Congreso de la República Declaran necesario uso del método de tamizaje para el diagnóstico del hipotiroidismo congénito. RM No 494-97-SA/DM. Lima: Congreso de la República; 1997.

5. Lu de Lama R. Tamizaje (Screening) neonatal del hipotiroidism congénito y enfermedades metabólicas. Rev Peru Pediatr. 2002;55(1):72-3.

6. Fukushi M, Hanai J, Yamaguchi A, Mikami A, Honma K, Nomura Y, et al. 
International cooperation in neonatal screening: technical training course for newborn and infant screening. Southeast Asian J Trop Med Public Health. 1999;30 Suppl 2:43-4.

7. Galan-Rodas E, Duenas M, Obando S, Saborio M. Tamizaje neonatal en el Perú: ¿hacia donde vamos?. Rev Peru Med Exp Salud Publica. 2013;30(4):724-5.

8. inmp.gob.pe [Internet]. Lima: Instituto Nacional Materno Perinatal [actualizado Octubre de 2014]. Disponible en: http://www.inmp.gob. pe/servicios/tamizaje-neonatal-en-elinmp/1427209501

9. Perú, Ministerio de Salud. Norma técnica de salud para la atencion integral de salud de la niña y del niño (NTS) No 040-MINSA/DGSP-V.01. Lima: MINSA; 2006.

10. LaFranchi S, Huang SA. Newborn thyroid disorders and screening. En: Lavin N, editor. Manual of endocrinology and metabolism. 4ta ed. Philadelphia: Lippincottt Williams \& Wilkins; 2009. p. 457-77.

11. Rastogi MV, LaFranchiSH. Congenital hypothyroidism. Orphanet J Rare Dis. 2010;5:17.

12. LaFranchi SH, Hanna CE, Krainz PL, Skeels MR, Miyahira RS, Sesser DE. Screening for congenital hypothyroidism with specimen collection at two time periods: results of the Northwest Regional Screening Program. Pediatrics. 1985;76(5):734-40.

13. Léger J, Olivieri A, Donaldson $M$, Torresani T, Krude H, van Vliet G, et al. European Society for Paediatric Endocrinology consensus guidelines on screening, diagnosis, and management of congenital hypothyroidism. J Clin Endocrinol Metab. 2014;99(2):36384. doi: 10.1210/jc.2013-1891.

14. LaFranchi SH. Approach to the diagnosis and treatment of neonatal hypothyroidism. J Clin Endocrinol Metab. 2011;96(10):2959-67. doi: 10.1210/jc.2011-1175.

15. Blanco-López A, Amarilla T, DorantesAlvarez LM. [Clinical and laboratory evaluation of patients with congenital hypothyroidism]. Bol Med Hosp Infant Mex. 1986;43(4):228-32. [Artículo en español]

16. Van Vliet G. Polak M. Thyroid disorders in infancy. En: Lifshitz F, editor. Pediatric endocrinology. Vol 2. 5th ed. New York: Informa Healthcare; 2007. p. 395-8.

17. Harris KB, Pass KA. Increase in congenital hypothyroidism in New York State and in the United States. Mol Genet Metab. 2007;91(3):268-77.

18. Rose SR, Section on Endocrinology and Committee on Genetics, Brown RS, Public Health Committee. Update of newborn screening and therapy for congenital hypothyroidism. Pediatrics. 2006;117(6):2290-303.

19. Borrajo GJ. Newborn screening in Latin America at the beginning of the 21st century. Journal of inherited metabolic disease. Aug 2007;30(4):466-481.

20. Rojas MI, Garmendia Lorena F, Solis-Rosas C, Chavéz E, Bonzán I.
Detección precoz de hipotiroidismo congénito: encuesta preliminar. Diagnostico (Perú). 1984;14(5):135-7.

21. inmp.gob.pe [Internet]. Lima: Instituto Nacional Materno Perinatal [actualizado Octubre de 2014]. Disponible en: http://www.inmp.gob. pe/servicios/programa/1427209159

22. Del Aguila Villar CM, Rojas Gabulli MI, Falen Boggio J. Evaluación clínica y diagnóstico precoz de hipotiroidismo congénito. Diagnostico (Perú). 1992;30(3/6):41-5.

23. Selva KA, Harper A, Downs A, Blasco PA, Lafranchi SH. Neurodevelopmental outcomes in congenital hypothyroidism: comparison of initial T4 dose and time to reach target T4 and TSH. J Pediatr. 2005;147(6):775-80.

24. Instituto Nacional de Estadística e Informática. Nacimientos registrados por año de inscripcion segun departamento 2005-2011 [Internet]. Lima: INEI; 2011 [citado el 11 de mayo de 2014]. Disponible en: http:// www.inei.gob.pe/estadisticas/indicetematico/poblacion-y-vivienda/

Correspondencia: Lina Huerta-Saenz

Dirección: 3243 E. Murdock, Ste 201. Wichita, KS 67208.

Teléfono: 011 (316) 500-8900

Correoelectrónico:lhuertasaenz@cmh.edu 